\title{
Tackle injury epidemiology and performance in rugby league - narrative synthesis
}

\author{
N Burger, $\mathbf{1}$ (iD PhD, B Jones, 1,3,4,5,6 iD PhD, S Hendricks, ${ }^{\mathbf{1 , 3}}$ iD \\ $\mathrm{PhD}$ \\ ${ }^{1}$ Division of Exercise Science and Sports Medicine, Department of \\ Human Biology, University of Cape Town, Rondebosch, South Africa \\ ${ }^{2}$ Department of Public \& Occupational Health and the EMGO Institute for \\ Health and Care Research, VU University Medical Center, Amsterdam, The \\ Netherlands \\ ${ }^{3}$ Carnegie Applied Rugby Research (CARR) Centre, Carnegie School of \\ Sport, Leeds Beckett University, Leeds, UK \\ ${ }^{4}$ Leeds Rhinos Rugby League Club, Leeds, UK \\ ${ }^{5}$ England Performance Unit, The Rugby Football League, Leeds, UK \\ ${ }^{6}$ School of Science and Technology, University of New England, NSW, \\ Australia
}

Corresponding author: N Burger (nicholas.burger@uct.ac.za)

Background: In rugby league (RL), the ability to repeatedly engage in the tackle, whether as a ball carrier or tackler, is essential for team success and player performance. It is also the leading cause of injury, with over $90 \%$ of total injuries occurring during the tackle in professional and amateur cohorts. To effectively reduce the risk of injury and optimise performance, establishing the extent of the 'problem', through injury surveillance or descriptive performance studies is required.

Objective: The purpose of this narrative synthesis was to systematically search and synthesise tackle injury epidemiology and tackle performance frequency in RL. To achieve this objective, a systematic review was conducted. Methods: The search was limited to English-only articles published between January 1995 and October 2018. Based on the search criteria, a total of 53 studies were found: 32 focused on tackle injury epidemiology (nine cases studies) and 21 focused on tackle frequency.

Results: In general, over 600 tackles may occur during an RL match. Tackle injury frequencies (both overall and time-loss injuries) ranged between $47 \%-94 \%$ at the professional level, and between $38 \%-96 \%$ for the lower levels of play. A greater proportion of injuries occurring in professional RL are severe time-loss injuries when compared to lower levels of play. Most time-loss and overall injuries occur to players who are tackled, i.e., ball carriers, across all levels of play.

Conclusion: This narrative synthesis will facilitate tackle injury prevention and performance research in $\mathrm{RL}$, and act as a reference document for coaches and practitioners.

Keywords: tackler, ball carrier, injury prevention, aetiology, tackling

\section{S Afr J Sports Med 2021;33:1-8. DOI: 10.17159/2078-516X/2021/v33i1a9313}

Rugby league (RL) is an intermittent, highintensity, collision-based team sport. ${ }^{[1-3]}$ During a typical match, played over the course of $60-80$ players physically engage each other to compete for ball
planutes and depending on the level of play, possession and territory. ${ }^{[4]}$ This dynamic and physical contest is known as the tackle and can occur up to 700 times during a match. ${ }^{[2]}$ As such, the ability to repeatedly engage in the tackle, whether as a ball carrier or tackler, is essential for team success and player performance. ${ }^{[5]}$ It is also the leading cause of injury, with over $90 \%$ of total injuries occurring during the tackle in some professional and amateur cohorts. ${ }^{[6,7]}$

To effectively reduce the risk of injury and optimise performance, following a sport injury prevention or sport performance process model such as the Translating Research into Injury Prevention Practice (TRIPP) model ${ }^{[8]}$ (involving six stages) or the Applied Research Model for the Sport Sciences (ARMSS) $^{[9]}$ (involving eight stages), is recommended. These models outline a sequence of stages that need to be completed to ensure the uptake and sustainability of an injury prevention or performance initiative in the 'real world' ${ }^{[8]}$ In these models, the first stage proposes establishing the extent of the 'problem' through injury surveillance or descriptive performance studies. The second stage aims to understand why and how injuries or performances occur, that is, identifying injury risk factors and performance determinants. The third stage seeks to develop potential preventive and performance enhancing measures for testing in ideal or controlled conditions (Stage four). Stage five aims to understand the implementation context and the final stage (Stage six) monitors the effectiveness of the preventive and performance measure in the real world.

The first stage is essential to these injury prevention and performance process models, as it provides direction and focus to the subsequent stages. A large proportion of RL injury and performance research falls within the first stage of the sequence. This has also shown to be the case in rugby union research. ${ }^{[10]}$ Literature reviews aim to search and synthesise this body of research with the goal of pushing forward the injury prevention and performance agenda, and to inform practice and coaching. While general epidemiology and performance reviews for RL have been conducted, ${ }^{[4,11]}$ a review specifically synthesising tackle injury epidemiology and tackle frequencies by playing levels, competition, and by role (ball carrier or tackler) has not been published. Therefore, to fulfil the first stage of TRIPP and AARMS, the purpose of this narrative synthesis was to systematically search and synthesise tackle injury epidemiology and tackle performance frequencies in RL.

\section{Methods}

\section{Search strategy}

A search was conducted for publications that reported tacklespecific factors in RL (rugby union and sevens were included in the search and analysed separately) ${ }^{[10]}$ according to systematic review guidelines. ${ }^{[12,13]}$ Three electronic databases (PubMed, Scopus and Web of Science) were searched using the keyword combinations 'rugby' AND 'contact', 'rugby' AND 'tackle', and 'rugby' AND ‘league' AND ‘injur”.

\section{Eligibility criteria}

The search was limited to English-only articles published 
between January 1995 and October 2018. Articles that involved quantitative data on RL (including all ages and levels of play, and male and female players) were included. Only studies that included tackle-related match and/or training data (pertaining to tacklers, and/or ball carriers) specific to (1) injury epidemiology and (2) tackle performance frequencies were selected for inclusion. The included studies clearly defined and included the tackle or ball carrying as part of their analysis and did not group these aspects of play into general contact/collision data thus rendering them indistinguishable from other contact phases.

Rates for overall and time-loss tackle injuries (only studies reporting number of injuries per 1000 hours of exposure) and tackle-related injury frequencies (percentages) were tabulated (Tables 1-2). A time-loss injury is defined as an injury that resulted in a player being absent from normal match/training/recreational activities for seven days more (this definition was used in all studies reported in the current analysis but may differ for other studies) after the incident. Medical attention injuries are injuries that required treatment from a healthcare professional but resulted in no time away from normal activities. Overall injuries include both medical attention and timeloss injuries events. Injury case studies specific to tackle events were also reviewed (see Appendix in the supplementary material). Overall tackle numbers and rates (only studies reporting total number of tackles per match, and/or number of tackles per game or per minute) were tabulated (Table 3) and included in the current study. Narrative literature reviews, systematic reviews, meta-analyses, editorials, journal letters, book chapters, conference proceedings, discussions and qualitative research studies were excluded from the analysis. All other quantitative study types and case studies were accepted for review.

\section{Screening process}

A five-step approach was followed to identify the final group of articles that would eventually be included in the final review (Figure 1). Two authors (NB and $\mathrm{SH}$ ) independently screened the titles using the eligibility criteria specified. The reliability of the authors was assessed by comparing the results of the titlescreening process. Disparities in the results were discussed and resolved by the authors. NB continued the screening process of the abstracts and full-text articles. The articles were excluded at each step if they met the exclusion criteria or did not meet the inclusion criteria.

\section{Data interpretation}

Only key findings (relating to tackle events) from each study were presented in this review. Confidence intervals (95\% CIs)

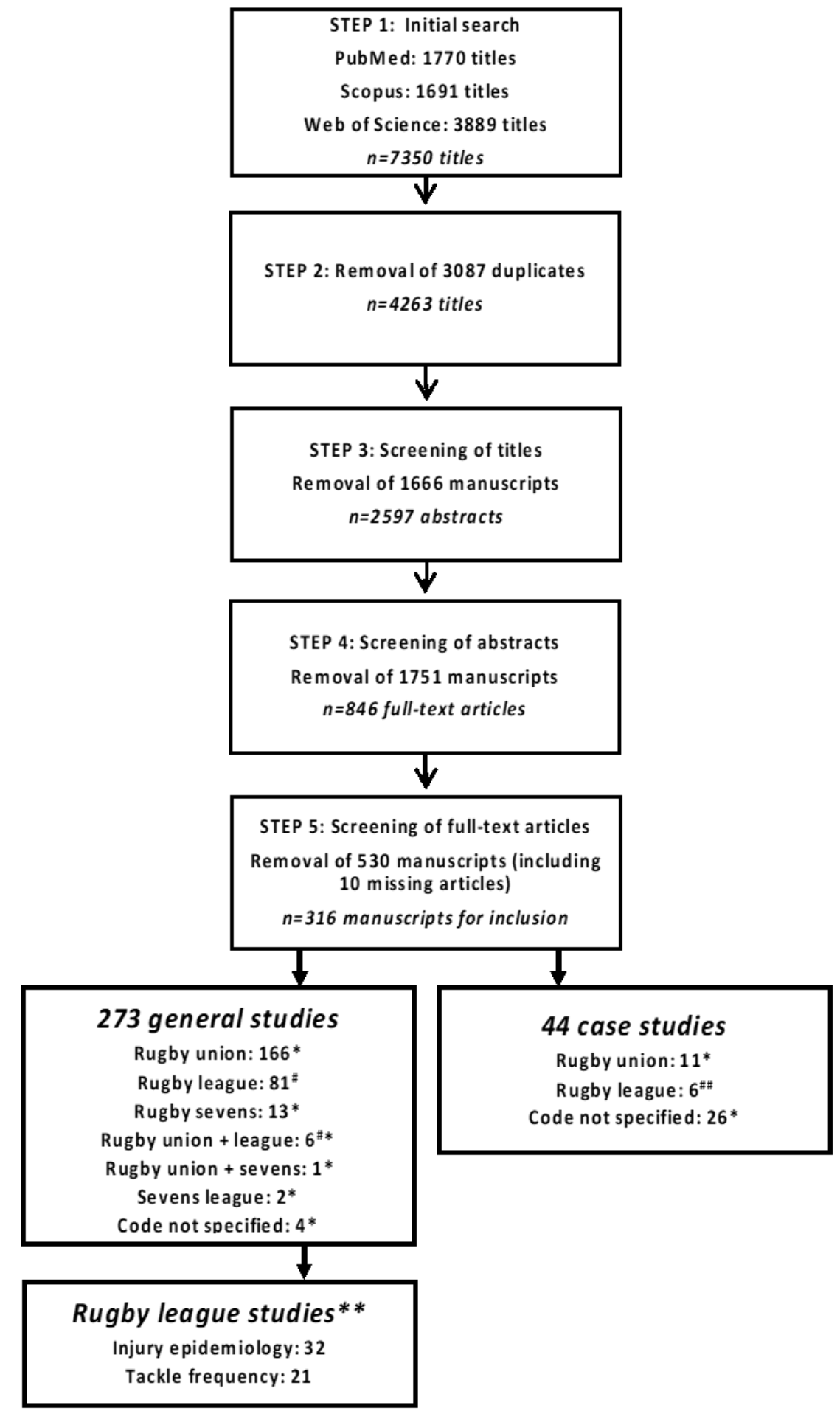

Fig. 1. Summary of the literature screening process. ${ }^{*}$, manuscripts included in RL analysis; \#, manuscripts included in RL appendix; *, manuscripts included in separate rugby union and sevens analysis/appendix; ${ }^{* *}, R L$ manuscripts included in the current study only. 
and standard deviations ( \pm SDs) were provided in the tables (depending on availability). All values were rounded off to a maximum of two decimal places, where necessary.

\section{Results}

The overall and time-loss injury frequencies and rates for professional RL are shown in Table 1 and all other levels in Table 2. Other RL tackle injury-related research also focused on specific types of injuries including concussion, ${ }^{[14-20]}$ spinal and neck injuries, ${ }^{[21]}$ and shoulder injuries. ${ }^{[22]}$ Tackle injury case-studies were also found and are summarised (see Appendix in the supplementary material).

The overall number of tackles per match and tackle rates (tackles per game or per minute) for all levels of play are shown in Table 3. Successful and unsuccessful tackles were grouped together.

\section{Discussion}

The aim of this narrative synthesis was to systematically search for tackle injury and tackle frequency performance studies in RL and synthesise these with the goal of facilitating tackle injury prevention and performance research. This can be regarded as a reference document for coaches and practitioners. Based on the search criteria, a total of 53 studies were found: 32 focused on tackle injury epidemiology (with a further nine cases studies) and 21 focused on tackle frequency. In general, over 600 tackles may occur during a RL match. Each forward executes between 20-25 tackles, which is double that of backs. In contrast, forwards and backs carry the ball into the tackle, i.e. being tackled, at similar frequencies. A major challenge with the current body of literature on tackle frequencies in RL is the lack of or inconsistencies in defining the tackle. Studies have either used the definition of the tackle based on RL law, when the ball carrier is held by one or more of the opposing players, and either the ball or hand of the arm holding the ball makes contact with the ground, or the ball carrier cannot make any further progress, or it is used as a definition where 'attempted' tackles are included. To improve the consistency and quality of tackle research in RL, a set of clear descriptions and definitions need to be developed and agreed upon, similar to those used in rugby union. [56]

Tackle injury frequencies (both overall and time-loss injuries) ranged between $47 \%-94 \%$ at the professional level and between $38 \%-96 \%$ for the lower levels of play. Up to $73 \%$ of time-loss injuries, i.e. more severe injuries resulting in the player being absent from matches or training sessions taking place at least one week after the initial injury event, occurred during the tackle at the professional level. ${ }^{[2]}$ This frequency was lower at the amateur level, with up to $61 \%$ of time-loss injuries occurring during tackles. ${ }^{[31]}$ Also, the majority of time-loss and overall injuries occur to the tackled player (i.e. the ball carrier) in professional RL (63\%) and across all other levels (61\%). King et al. ${ }^{[6]}$ reported a high overall tackle-related injury incidence in a National Rugby League cohort (300 injuries per 1000 hours). This rate is substantially higher than the other figures reported in professional RL which were estimated at around 77.4 injuries per 1000 hours. ${ }^{[2]}$ However, overall injury rates are substantially higher in semi-professional cohorts, reaching up to 382.2 injuries per 1000 hours, ${ }^{[32]}$ and are even greater in amateur RL, with one study reporting incidences as high as 541 injuries per 1000 hours. ${ }^{[39]}$ It is worth noting that the majority of injury studies were conducted on single teams, which may explain why injury frequencies and incidences varied between studies. Although there were no explicit variations in the methods used to collect injury data across the studies, the differences in incidence rates may also be attributed to

Table 1. Tackle-related injury frequencies and rates in professional Rugby League

\begin{tabular}{|c|c|c|c|c|}
\hline Study & Cohort & Injury definition & Injury frequency & Injury rate \\
\hline $\begin{array}{l}\text { Gissane et al., } \\
1998[23]\end{array}$ & $\begin{array}{l}\text { European Super } \\
\text { League }\end{array}$ & $\begin{array}{l}\text { Time-loss } \\
\text { (>7 days) }\end{array}$ & $\begin{array}{l}70 \% \text { (35\% tackled, } \\
35 \% \text { tackling) }\end{array}$ & $\begin{array}{l}35.2 / 1000 \text { hours }(17.6 / 1000 \text { hours tackled; } 17.6 / 1000 \\
\text { hours tackling) }\end{array}$ \\
\hline $\begin{array}{l}\text { Gissane et al., } \\
\text { 2003[24] }\end{array}$ & $\begin{array}{l}\text { European Super } \\
\text { League }\end{array}$ & $\begin{array}{l}\text { Time-loss } \\
\text { (>7 days) }\end{array}$ & $\begin{array}{l}73 \% \text { (50\% tackled, } \\
23 \% \text { tackling) }\end{array}$ & $\begin{array}{l}\text { 43.8/1000 hours }(30.1 / 1000 \text { hours tackled }[95 \% \text { CI } 23.7- \\
37.8] ; 13.7 / 1000 \text { hours tackling [95\%CI 9.5-19.1]) }\end{array}$ \\
\hline $\begin{array}{l}\text { Gissane et al., } \\
\text { 2003[25] }\end{array}$ & $\begin{array}{l}\text { European Super } \\
\text { League }\end{array}$ & $\begin{array}{l}\text { Overall and time- } \\
\text { loss ( }>1 \text { day) }\end{array}$ & $\begin{array}{l}74 \% \text { ( } 44 \% \text { tackled, } \\
30 \% \text { tackling) }\end{array}$ & N/A \\
\hline $\begin{array}{l}\text { Gabbett et al., } \\
2012^{[26]}\end{array}$ & $\begin{array}{l}\text { National Rugby } \\
\text { League }\end{array}$ & $\begin{array}{l}\text { Overall and time- } \\
\text { loss ( }>1 \text { day) }\end{array}$ & N/A & $\begin{array}{l}\text { 77.4/1000 hours (30/1000 hours tackled [95\%CI 22.6- } \\
\text { 39.7]; 47.4/1000 hours tackling [95\%CI 38.0-59.1]) }\end{array}$ \\
\hline $\begin{array}{l}\text { Georgeson et al., } \\
2012^{[27]}\end{array}$ & $\begin{array}{l}\text { National Rugby } \\
\text { League }\end{array}$ & $\begin{array}{l}\text { Overall and time- } \\
\text { loss ( }>1 \text { day) }\end{array}$ & $\begin{array}{l}49 \% \text { ( } 41 \% \text { tackled, } \\
8 \% \text { tackling) }\end{array}$ & $\mathrm{N} / \mathrm{A}$ \\
\hline $\begin{array}{l}\text { King et al., } \\
2012^{[6]}\end{array}$ & $\begin{array}{l}\text { National Rugby } \\
\text { League }\end{array}$ & $\begin{array}{l}\text { Overall and time- } \\
\text { loss ( }>7 \text { days })\end{array}$ & $\begin{array}{l}94 \% \text { (50\% tackled, } \\
44 \% \text { tackling) }\end{array}$ & $300 / 1000$ hours (95\%CI 265-340) \\
\hline $\begin{array}{l}\text { Sanctuary et al., } \\
2012^{[28]}\end{array}$ & $\begin{array}{l}\text { European Super } \\
\text { League }\end{array}$ & $\begin{array}{l}\text { Overall and time- } \\
\text { loss ( }>1 \text { day) }\end{array}$ & $72 \%$ & N/A \\
\hline $\begin{array}{l}\text { Ullah et al., } \\
2014[29]\end{array}$ & $\begin{array}{l}\text { National Rugby } \\
\text { League }\end{array}$ & $\begin{array}{l}\text { Overall and time- } \\
\text { loss ( }>1 \text { day) }\end{array}$ & $47 \%$ & $\mathrm{~N} / \mathrm{A}$ \\
\hline
\end{tabular}

N/A, This data is not available for the study 
Table 2. Tackle-related injury frequencies and rates in semi-professional, amateur and youth Rugby League

\begin{tabular}{|c|c|c|c|c|}
\hline Study & Cohort & Injury definition & Injury frequency & Injury rate \\
\hline Babic et al., 2001[30] & $\begin{array}{l}\text { Croatian-Slovenian } \\
\text { amateur rugby league }\end{array}$ & $\begin{array}{l}\text { Overall and time- } \\
\text { loss ( }>1 \text { day) }\end{array}$ & $\begin{array}{l}55 \%(25 \% \text { tackled, } 30 \% \\
\text { tackling) }\end{array}$ & N/A \\
\hline Gabbett, 2001[31] & $\begin{array}{l}\text { Second Division amateur } \\
\text { rugby league }\end{array}$ & $\begin{array}{l}\text { Time-loss (>7 } \\
\text { days) }\end{array}$ & $\begin{array}{l}61 \%(35 \% \text { tackled, } 26 \% \\
\text { tackling) }\end{array}$ & 22.8/1000 hours (95\%CI 12.0-33.7) \\
\hline Gabbett, 2003[32] & $\begin{array}{l}\text { Australian semi- } \\
\text { professional rugby league }\end{array}$ & $\begin{array}{l}\text { Overall and time- } \\
\text { loss (>1 day) }\end{array}$ & $46 \%$ & $382.2 / 1000$ hours (95\%CI 355.5-408.9) \\
\hline Gabbett, 2004[33] & $\begin{array}{l}\text { Australian semi- } \\
\text { professional rugby league }\end{array}$ & $\begin{array}{l}\text { Overall and time- } \\
\text { loss (>1 day) }\end{array}$ & $\begin{array}{l}38 \%(22 \% \text { tackled, } 16 \% \\
\text { tackling) }\end{array}$ & $\begin{array}{l}\text { 350.2/1000 hours (202.2/1000 hours tackled } \\
\text { [95\%CI } 174.8-229.7] ; 148.0 / 1000 \text { hours } \\
\text { tackling }[95 \% \text { CI } 124.5-171.6])\end{array}$ \\
\hline Gabbett, 2005[34] & $\begin{array}{l}\text { Australian semi- } \\
\text { professional rugby league }\end{array}$ & $\begin{array}{l}\text { Time-loss (>7 } \\
\text { days) }\end{array}$ & $53 \%$ & $\begin{array}{l}36.0 / 1000 \text { hours }(19.0 / 1000 \text { hours tackled } \\
{[95 \% \text { CI } 13.0-24.0] ; 17.0 / 1000 \text { hours tackling }} \\
[95 \% \text { CI } 11.0-23.0])\end{array}$ \\
\hline Gabbett, 2005[35] & $\begin{array}{l}\text { Sub-elite rugby league } \\
\left({ }^{*} \text { unlimited interchange }\right)\end{array}$ & $\begin{array}{l}\text { Time-loss (>7 } \\
\text { days) }\end{array}$ & N/A & $\begin{array}{l}\text { 37.4/1000 hours (19.8/1000 hours tackled } \\
{[95 \% \text { CI } 12.3-27.2] ; 17.6 / 1000 \text { hours tackling }} \\
[95 \% \text { CI } 10.5-24.6])\end{array}$ \\
\hline Gabbett, 2005[35] & $\begin{array}{l}\text { Sub-elite rugby league } \\
\left({ }^{*} \text { limited interchange }\right)\end{array}$ & $\begin{array}{l}\text { Time-loss }(>7 \\
\text { days) }\end{array}$ & $\mathrm{N} / \mathrm{A}$ & $\begin{array}{l}\text { 37.5/1000 hours (24.0/1000 hours tackled } \\
{[95 \% \text { CI } 12.2-35.7] ; 13.5 / 1000 \text { hours tackling }} \\
[95 \% \text { CI } 4.7-22.3])\end{array}$ \\
\hline $\begin{array}{l}\text { Gabbett and } \\
\text { Domrow, 2005[36] }\end{array}$ & $\begin{array}{l}\text { Australian semi- } \\
\text { professional rugby league }\end{array}$ & $\begin{array}{l}\text { Time-loss (>7 } \\
\text { days) }\end{array}$ & $\begin{array}{l}54 \% \text { (30\% tackled, } 24 \% \\
\text { tackling) }\end{array}$ & $\begin{array}{l}29.7 / 1000 \text { hours }(16.5 / 1000 \text { hours tackled } \\
{[95 \% \text { CI } 12.1-20.9] ; 13.2 / 1000 \text { hours tackling }} \\
{[95 \% \text { CI 9.3-17.1]) }}\end{array}$ \\
\hline King et al., 2006[37] & $\begin{array}{l}\text { New Zealand semi- } \\
\text { professional rugby league }\end{array}$ & $\begin{array}{l}\text { Overall and time- } \\
\text { loss ( }>1 \text { day) }\end{array}$ & $\begin{array}{l}66 \% \text { ( } 43 \% \text { tackled, } 23 \% \\
\text { tackling) }\end{array}$ & $\begin{array}{l}242.7 / 1000 \text { hours }(158.3 / 1000 \text { hours tackled } \\
{[95 \% \text { CI } 78.2-238.4] ; 84.4 / 1000 \text { hours tackling }} \\
[95 \% \text { CI } 25.9-142.9])\end{array}$ \\
\hline King et al., 2006 [37] & $\begin{array}{l}\text { New Zealand amateur } \\
\text { rugby league amateur }\end{array}$ & $\begin{array}{l}\text { Overall and time- } \\
\text { loss ( }>1 \text { day) }\end{array}$ & $\begin{array}{l}68 \%(44 \% \text { tackled, } 24 \% \\
\text { tackling) }\end{array}$ & $\begin{array}{l}483.1 / 1000 \text { hours }(310.6 / 1000 \text { hours tackled } \\
\text { [95\%CI } 167.1-454.0] ; 172.5 / 1000 \text { hours } \\
\text { tackling }[95 \% \text { CI } 65.6-279.5])\end{array}$ \\
\hline Gabbett, 2008[38] & Under-19 rugby league & $\begin{array}{l}\text { Time-loss (>7 } \\
\text { days) }\end{array}$ & N/A & $\begin{array}{l}\text { 29.3/1000 hours (19.2/1000 hours tackled } \\
{[95 \% \text { CI } 11.0-27.5] ; 10.1 / 1000 \text { hours tackling }} \\
[95 \% \text { CI } 4.1-16.0])\end{array}$ \\
\hline King et al., 2009[39] & $\begin{array}{l}\text { Division } 1 \text { Premier Grade } \\
\text { amateur rugby league }\end{array}$ & $\begin{array}{l}\text { Overall and time- } \\
\text { loss ( }>1 \text { day) }\end{array}$ & $\begin{array}{l}86 \% \text { (overall) }(48 \% \\
\text { tackled, } 37 \% \text { tackling); } \\
87 \% \text { (time-loss) }(51 \% \\
\text { tackled, } 36 \% \text { tackling) }\end{array}$ & $\begin{array}{l}\text { 228.1/1000 hours (overall) }(128.5 / 1000 \text { hours } \\
\text { tackled [95\%CI 103.2-160.0]; } 99.6 / 1000 \text { hours } \\
\text { tackling [95\%CI } 77.7-127.8]) ; 54.6 / 1000 \text { hours } \\
\text { (time-loss) }(32.1 / 1000 \text { hours tackled [95\%CI } \\
\text { 20.7-49.8]; } 22.5 / 1000 \text { hours tackling [ } 95 \% \mathrm{CI} \\
13.3-38.0] \text { ) }\end{array}$ \\
\hline King et al., 2009[39] & $\begin{array}{l}\text { Division } 2 \text { Premier Grade } \\
\text { amateur rugby league }\end{array}$ & $\begin{array}{l}\text { Overall and time- } \\
\text { loss (>1 day) }\end{array}$ & $\begin{array}{l}77 \% \text { (overall) }(58 \% \\
\text { tackled, } 19 \% \text { tackling); } \\
71 \% \text { (time-loss) }(44 \% \\
\text { tackled, } 27 \% \text { tackling) }\end{array}$ & $\begin{array}{l}\text { 541.0/1000 hours (overall) }(404.9 / 1000 \text { hours } \\
\text { tackled [95\%CI 338.3-484.5]; } 136.1 / 1000 \\
\text { hours tackling [95\%CI } 99.8-185.5]) ; \\
\text { 153.1/1000 hours (time-loss) }(95.3 / 1000 \text { hours } \\
\text { tackled [ } 95 \% \text { CI } 65.8-138.0] ; 57.8 / 1000 \text { hours } \\
\text { tackling [95\%CI 36.0-93.0]) }\end{array}$ \\
\hline King et al., 2010[7] & $\begin{array}{l}\text { Premier division amateur } \\
\text { rugby league }\end{array}$ & $\begin{array}{l}\text { Overall and time- } \\
\text { loss ( }>1 \text { day) }\end{array}$ & $\begin{array}{l}96 \%(68 \% \text { tackled, } 28 \% \\
\text { tackling) }\end{array}$ & $\begin{array}{l}\text { 501.3/1000 hours }(356.7 / 1000 \text { hours tackled } \\
\text { [95\%CI } 258.4-492.3] ; 144.6 / 1000 \text { hours } \\
\text { tackling [95\%CI 87.2-239.8]) }\end{array}$ \\
\hline $\begin{array}{l}\text { Orr and Cheng, } \\
2016^{40]}\end{array}$ & $\begin{array}{l}\text { Elite Australian junior } \\
\text { rugby league }\end{array}$ & $\begin{array}{l}\text { Overall and time- } \\
\text { loss ( }>1 \text { day) }\end{array}$ & $\begin{array}{l}61 \% \text { (overall) }(36 \% \\
\text { tackled, } 25 \% \text { tackling); } \\
66 \% \text { (time-loss) }(42 \% \\
\text { tackled, } 24 \% \text { tackling) }\end{array}$ & N/A \\
\hline
\end{tabular}

N/A, This data is not available for the study

discrepancies in the levels of efficiency between the individual data capturers, although this cannot be confirmed.

This narrative synthesis was limited to the first stage of the TRIPP and AARMS models. As such, RL studies that are tackle-related but did not meet the criteria were excluded. For example, studies that focused on describing the types of tackles, ${ }^{[2,48]}$ tackle technique, ${ }^{[5,57-62]}$ or the physiological and physical loads associated with the tackle, ${ }^{[43,47,48,51,52]}$ were not included in the current review. Arguably, these studies relate to why and how injuries occur or what is required to succeed in the tackle, which fulfils the objectives of the second stage in the TRIPP and AARMS models, i.e. identifying injury risk factors and performance determinants. While an analysis of all tackle injury risk factors and performance determinants in RL was beyond the scope of the current synthesis, this paper forms the basis for such a review. 
Table 3. Tackle numbers and rates in Rugby League (all levels of play)

\begin{tabular}{|c|c|c|c|}
\hline Study & Cohort & Rate definition & Tackle rate \\
\hline $\begin{array}{l}\text { Sirotic et al., } \\
20099^{[41]}\end{array}$ & $\begin{array}{l}\text { National Rugby } \\
\text { League }\end{array}$ & Tackling/minute & $0.25(\mathrm{SD} \pm 0.16)\left(1^{\text {st }}\right.$ half: 0.26 [SD \pm 0.18$] ; 2^{\text {nd }}$ half: $\left.0.23[\mathrm{SD} \pm 0.16]\right)$ \\
\hline $\begin{array}{l}\text { Sirotic et al., } \\
2009^{[41]}\end{array}$ & $\begin{array}{l}\text { Semi-professional } \\
\text { New South Wales } \\
\text { Premier League }\end{array}$ & Tackling/minute & 0.28 (SD \pm 0.16$)$ (1 $1^{\text {st }}$ half: $0.30[\mathrm{SD} \pm 0.17] ; 2^{\text {nd }}$ half: $\left.0.27[\mathrm{SD} \pm 0.16]\right)$ \\
\hline $\begin{array}{l}\text { King et al., } \\
2010^{[2]}\end{array}$ & $\begin{array}{l}\text { Elite rugby league } \\
\text { (international, NRL, } \\
\text { national youth } \\
\text { competition) }\end{array}$ & $\begin{array}{l}\text { Total match tackles } \\
\text { (tackling and tackled) } \\
\text { Tackling/game } \\
\text { Tackled/game }\end{array}$ & $\begin{array}{l}701(\mathrm{SD} \pm 64)^{\mathrm{a}} \\
32(\mathrm{SD} \pm 15)^{\mathrm{b}} \\
21(\mathrm{SD} \pm 4)^{\mathrm{c}}\end{array}$ \\
\hline $\begin{array}{l}\text { Gabbett et al., } \\
2011^{[42]}\end{array}$ & $\begin{array}{l}\text { National Rugby } \\
\text { League }\end{array}$ & $\begin{array}{l}\text { Total match tackling attempts } \\
\text { Tackling/game }\end{array}$ & $\begin{array}{l}265.1(\mathrm{SD} \pm 214.1)^{\mathrm{a}} \\
17.1(\mathrm{SD} \pm 9.1)^{\mathrm{b}}\end{array}$ \\
\hline $\begin{array}{l}\text { McLellan et al., } \\
\text { 2011 }\end{array}$ & $\begin{array}{l}\text { National Rugby } \\
\text { League }\end{array}$ & $\begin{array}{l}\text { Tackling/game } \\
\text { Tackled/game }\end{array}$ & $\begin{array}{l}\left.14.9(\mathrm{SD} \pm 10.5)^{\mathrm{a}} \text { (forwards: } 20.1[\mathrm{SD} \pm 11.3] ; \text { backs: } 10.7[\mathrm{SD} \pm 8.0]\right) \\
\left.10.2(\mathrm{SD} \pm 3.8)^{\mathrm{b}} \text { (forwards: } 10.9[\mathrm{SD} \pm 4.2] ; \text { backs: } 9.7[\mathrm{SD} \pm 3.5]\right)\end{array}$ \\
\hline $\begin{array}{l}\text { Sirotic et al., } \\
2011^{[44]}\end{array}$ & $\begin{array}{l}\text { National Rugby } \\
\text { League }\end{array}$ & Tackling/minute & $\begin{array}{l}\text { Backs: } 0.12(\mathrm{SD} \pm 0.09) ; \text { Forwards: } 0.41(\mathrm{SD} \pm 0.07) \\
\text { Fullbacks: } 0.05(\mathrm{SD} \pm 0.02) \text {; Hookers: } 0.34(\mathrm{SD} \pm 0.11) \\
\text { Service: } 0.31(\mathrm{SD} \pm 0.12)\end{array}$ \\
\hline $\begin{array}{l}\text { Sykes et al., } \\
2011^{[45]}\end{array}$ & $\begin{array}{l}\text { English Super League } \\
\text { and National Rugby } \\
\text { League }\end{array}$ & $\begin{array}{l}\text { Tackling/minute } \\
\text { Tackled/minute }\end{array}$ & $\begin{array}{l}1^{\text {st }} \text { quarter: } 0.28(\mathrm{SD} \pm 0.21)^{\mathrm{a}} ; 0.14(\mathrm{SD} \pm 0.10)^{\mathrm{b}} \\
2^{\text {nd }} \text { quarter: } 0.28(\mathrm{SD} \pm 0.27)^{\mathrm{a}} ; 0.13(\mathrm{SD} \pm 0.12)^{\mathrm{b}} \\
3^{\text {rd }} \text { quarter: } 0.26(\mathrm{SD} \pm 0.22)^{\mathrm{a}} ; 0.13(\mathrm{SD} \pm 0.10)^{\mathrm{b}} \\
4^{\text {th }} \text { quarter: } 0.32(\mathrm{SD} \pm 0.33)^{\mathrm{a}} ; 0.14(\mathrm{SD} \pm 0.09)^{\mathrm{b}} \\
\end{array}$ \\
\hline $\begin{array}{l}\text { Duffield et al., } \\
2012^{[46]}\end{array}$ & $\begin{array}{l}\text { Amateur rugby } \\
\text { league }\end{array}$ & $\begin{array}{l}\text { Tackles (tackling and } \\
\text { tackled)/game }\end{array}$ & $22(\mathrm{SD} \pm 9)$ \\
\hline $\begin{array}{l}\text { King et al., } \\
2012^{[6]}\end{array}$ & $\begin{array}{l}\text { Professional rugby } \\
\text { league }\end{array}$ & $\begin{array}{l}\text { Total match tackles } \\
\text { (tackling and tackled) }\end{array}$ & 657 \\
\hline $\begin{array}{l}\text { McLellan and } \\
\text { Lovell, 2012[47] }\end{array}$ & $\begin{array}{l}\text { National Rugby } \\
\text { League }\end{array}$ & $\begin{array}{l}\text { Tackling/game } \\
\text { Tackled/game }\end{array}$ & $\begin{array}{l}19.9(\mathrm{SD} \pm 10.5)^{\mathrm{a}}(\text { forwards: } 26.1[\mathrm{SD} \pm 15.3] ; \text { backs: } 10.7[\mathrm{SD} \pm 8.9]) \\
12.2(\mathrm{SD} \pm 3.6)^{\mathrm{b}}(\text { forwards: } 13.8[\mathrm{SD} \pm 5.2] ; \text { backs: } 11.7[\mathrm{SD} \pm 4.6])\end{array}$ \\
\hline $\begin{array}{l}\text { Twist et al., } \\
2012^{[48]}\end{array}$ & $\begin{array}{l}\text { European Super } \\
\text { League }\end{array}$ & $\begin{array}{l}\text { Tackling/game } \\
\text { Tackling/minute } \\
\text { Tackled/game } \\
\text { Tackled/minute }\end{array}$ & $\begin{array}{l}\text { Forwards: } 25.5(\mathrm{SD} \pm 13.7)^{\mathrm{a}} ; 0.5(\mathrm{SD} \pm 0.2)^{\mathrm{b}} ; 12.7(\mathrm{SD} \pm 6.1)^{\mathrm{c}} ; 0.3 \\
(\mathrm{SD} \pm 0.1)^{\mathrm{d}} \\
\text { Backs: } 13.6(\mathrm{SD} \pm 7.9)^{\mathrm{a}} ; 0.2(\mathrm{SD} \pm 0.1)^{\mathrm{b}} ; 11.6(\mathrm{SD} \pm 3.4)^{\mathrm{c}} ; 0.1(\mathrm{SD} \pm 0.04)^{\mathrm{d}}\end{array}$ \\
\hline $\begin{array}{l}\text { Johnston et al., } \\
20133^{[49]}\end{array}$ & $\begin{array}{l}\text { Amateur rugby } \\
\text { league }\end{array}$ & Tackling/minute & $0.3(\mathrm{SD} \pm 0.1)$ \\
\hline $\begin{array}{l}\text { McGuckin et al., } \\
\text { 2014[50] }\end{array}$ & $\begin{array}{l}\text { National Rugby } \\
\text { League }\end{array}$ & Tackling/game & Home games: 14.3 (SD \pm 6.7$)$; away games: $18.4(\mathrm{SD} \pm 9.2)$ \\
\hline $\begin{array}{l}\text { Cummins and } \\
\text { Orr, 2015[51] }\end{array}$ & $\begin{array}{l}\text { National Rugby } \\
\text { League }\end{array}$ & $\begin{array}{l}\text { Tackling/game } \\
\text { Tackling/minute } \\
\text { Tackled/game } \\
\text { Tackled/minute }\end{array}$ & $\begin{array}{l}\text { Hit-up forwards: } 21.5(\mathrm{SD} \pm 6.0)^{\mathrm{a}} ; 0.53(\mathrm{SD} \pm 0.08)^{\mathrm{b}} ; 9.0(\mathrm{SD} \pm 3.8)^{\mathrm{c}} \text {; } \\
0.20(\mathrm{SD} \pm 0.04)^{\mathrm{d}} \\
\text { Wide-running forwards: } 20.5(\mathrm{SD} \pm 5.0)^{\mathrm{a}} ; 0.39(\mathrm{SD} \pm 0.10)^{\mathrm{b}} ; 8.0 \\
(\mathrm{SD} \pm 3.6)^{\mathrm{c}} ; 0.20(\mathrm{SD} \pm 0.10)^{\mathrm{d}} \\
\text { Adjustables: } 17.0(\mathrm{SD} \pm 12.5)^{\mathrm{a}} ; 0.41(\mathrm{SD} \pm 0.20)^{\mathrm{b}} ; 5.0(\mathrm{SD} \pm 4.5)^{\mathrm{c}} ; 0.1 \\
(\mathrm{SD} \pm 0.00)^{\mathrm{d}} \\
\text { Outside backs: } 7.0(\mathrm{SD} \pm 6.0)^{\mathrm{a}} ; 0.08(\mathrm{SD} \pm 0.07)^{\mathrm{b}} ; 11.2(\mathrm{SD} \pm 2.1)^{\mathrm{c}} ; 0.1 \\
(\mathrm{SD} \pm 0.02)^{\mathrm{d}}\end{array}$ \\
\hline $\begin{array}{l}\text { Fletcher et al., } \\
\text { 2015[52] }\end{array}$ & $\begin{array}{l}\text { European Super } \\
\text { League }\end{array}$ & $\begin{array}{l}\text { Tackling/game } \\
\text { Tackled/game }\end{array}$ & $\begin{array}{l}\text { Forwards: } 24(\mathrm{SD} \pm 13)^{\mathrm{a}} ; 8.5(\mathrm{SD} \pm 5)^{\mathrm{b}} ; \text { Backs: } 8(\mathrm{SD} \pm 10)^{\mathrm{a}} ; 9(\mathrm{SD} \pm 4)^{\mathrm{b}} \\
\text { Adjustables: } 14(\mathrm{SD} \pm 12)^{\mathrm{a}} ; 4(\mathrm{SD} \pm 4)^{\mathrm{b}}\end{array}$ \\
\hline $\begin{array}{l}\text { Speranza et al., } \\
\text { 2015[53] }\end{array}$ & $\begin{array}{l}\text { Semi-professional } \\
\text { rugby league }\end{array}$ & Tackling/game & Forwards: 24.3 (SD \pm 6.5$)$; Backs: 13.2 (SD \pm 8.5$)$ \\
\hline $\begin{array}{l}\text { Gardner et al., } \\
2017^{[17]}\end{array}$ & $\begin{array}{l}\text { National Rugby } \\
\text { League }\end{array}$ & $\begin{array}{l}\text { Total match tackles } \\
\text { (tackling and tackled) }\end{array}$ & 632.1 \\
\hline $\begin{array}{l}\text { Dempsey et al., } \\
2018^{[54]}\end{array}$ & $\begin{array}{l}\text { Senior international } \\
\text { male rugby league }\end{array}$ & $\begin{array}{l}\text { Tackling/game } \\
\text { Tackling/minute } \\
\text { Tackled/game } \\
\text { Tackled/minute } \\
\end{array}$ & $\begin{array}{l}\text { Forwards: } 25.5(\mathrm{SD} \pm 8.4)^{\mathrm{a}} ; 0.47(\mathrm{SD} \pm 0.23)^{\mathrm{b}} ; 10.5(\mathrm{SD} \pm 3.6)^{\mathrm{c}} ; 0.20 \\
(\mathrm{SD} \pm 0.10)^{\mathrm{d}} \\
\text { Backs: } 13.4(\mathrm{SD} \pm 9.5)^{\mathrm{a}} ; 0.16(\mathrm{SD} \pm 0.11)^{\mathrm{b}} ; 11.9(\mathrm{SD} \pm 5.2)^{\mathrm{c}} ; 0.15 \\
(\mathrm{SD} \pm 0.08)^{\mathrm{d}}\end{array}$ \\
\hline $\begin{array}{l}\text { Dempsey et al., } \\
\text { 2018[54] }\end{array}$ & $\begin{array}{l}\text { Junior international } \\
\text { male rugby league }\end{array}$ & $\begin{array}{l}\text { Tackling/game } \\
\text { Tackling/minute } \\
\text { Tackled/game } \\
\text { Tackled/minute }\end{array}$ & $\begin{array}{l}\text { Forwards: } 19.2(\mathrm{SD} \pm 10.0)^{\mathrm{a}} ; 0.34(\mathrm{SD} \pm 0.13)^{\mathrm{b}} ; 6.5(\mathrm{SD} \pm 3.5)^{\mathrm{c}} ; 0.12 \\
(\mathrm{SD} \pm 0.06)^{\mathrm{d}} \\
\text { Backs: } 10.0(\mathrm{SD} \pm 6.7)^{\mathrm{a}} ; 0.13(\mathrm{SD} \pm 0.08)^{\mathrm{b}} ; 5.3(\mathrm{SD} \pm 3.5)^{\mathrm{c}} ; 0.06 \\
(\mathrm{SD} \pm 0.04)^{\mathrm{d}}\end{array}$ \\
\hline $\begin{array}{l}\text { Woods et al., } \\
20188^{[55]}\end{array}$ & $\begin{array}{l}\text { National Rugby } \\
\text { League }\end{array}$ & Total match tackle attempts & $325.0(\mathrm{SD} \pm 39.7)$ \\
\hline $\begin{array}{l}\text { Woods et al., } \\
\text { 2018 }\end{array}$ & Under-20 Holden Cup & Total match tackle attempts & $283.4(\mathrm{SD} \pm 35.6)$ \\
\hline
\end{tabular}




\section{Conclusion}

This review synthesises tackle injury and tackle frequency performance studies in RL, with the goal of facilitating tackle injury prevention and performance research, and acting as a reference document for coaches and practitioners. Based on the search criteria, a total of 53 studies were found: 32 focused on tackle injury epidemiology and 21 focused on tackle frequency. In general, over 600 tackles may occur during a RL match. Each forward executes about 20-25 tackles, which is double that of backs. In contrast, forwards and backs carry the ball into the tackle, i.e. being tackled, at similar frequencies. Tackle injury frequencies (both overall and time-loss injuries) ranged between $47 \%-94 \%$ at the professional level, and between $38 \%-96 \%$ for the lower levels of play. A greater proportion of severe time-loss injuries occur during the tackle at the professional level in comparison to lower levels of play. Furthermore, most time-loss and overall injuries occur to players who are tackled, i.e. ball carriers, across all levels of play.

Conflict of interest and source of funding: The authors declare no conflict of interest and no source of funding.

\section{Author contributions:}

NB and SH made significant contributions to the conceptualisation, design and drafting of the manuscript as part of NB's PhD thesis. NB, SH and BJ all contributed to the analysis and interpretation of the data as well as making major revisions. NB, SH and BJ all approved the final version to be published.

\section{References}

1. Gabbett TJ, Jenkins DG, Abernethy B. Physical collisions and injury in professional rugby league match-play. J Sci Med Sport 2011;14(3):210-215. [doi: 10.1016/j.jsams.2011.01.002] [PMID: 21324742]

2. King D, Hume PA, Clark T. Video analysis of tackles in professional rugby league matches by player position, tackle height and tackle location. Int J Perform Anal Sport 2010;10(3):241-254. [doi: 10.1080/24748668.2010.11868519]

3. Gabbett TJ. Physiological and anthropometric characteristics of amateur rugby league players. Br J Sports Med 2000;34(4):303-307. [doi: 10.1136/bjsm.34.4.303][PMID: 10953906]

4. Gabbett TJ. Science of rugby league football: a review. J Sports Sci 2005;23(9):961-976. [doi: 10.1080/02640410400023381] [PMID: 16195048]

5. Gabbett TJ. Influence of fatigue on tackling technique in rugby league players. J Strength Cond Res 2008;22(2):625632. [doi: 10.1519/JSC.0b013e3181635a6a][PMID: 18550983]

6. King D, Hume PA, Clark T. Nature of tackles that result in injury in professional rugby league. Res Sports Med 2012;20(2):86-104. [doi: 10.1080/15438627.2012.660824] [PMID: 22458826]

7. King D, Clark T, Kellmann M. Changes in stress and recovery as a result of participating in a premier rugby league representative competition. Int J Sports Sci Coach 2010;5(2):223-237. [doi: 10.1260/1747-9541.5.2.223]

8. Finch C. A new framework for research leading to sports injury prevention. J Sci Med Sport 2006;9(1-2):3-9. [doi: 10.1016/j.jsams.2006.02.009][PMID: 16616614]
9. Bishop D. An applied research model for the sport sciences. Sports Med 2008;38(3):253-263. [doi: 10.2165/00007256200838030-00005][PMID: 18278985]

10. Burger N, Lambert M, Hendricks S. Lay of the land: narrative synthesis of tackle research in rugby union and rugby sevens. BMJ Open Sport Exerc Med 2020;6(1):e000645. [doi: 10.1136/bmjsem-2019-000645][PMID: 32518671]

11. Booth M, Orr R, Cobley S. The effect of training loads on performance measures and injury characteristics in rugby league players. A systematic review. Int J Sports Physiol Perform 2018;13(10):1259-1272. [doi: 10.1123/ijspp.20170329][PMID: 29252061]

12. Popay J, Roberts H, Sowden A, et al. Guidance on the conduct of narrative synthesis in systematic reviews: A product from the ESRC Methods Programme. Lancaster Lancaster Univ. 2006;10(2.1):1018-4643. [doi: 10.13140/2.1.1018.4643]

13. Druetz T, Siekmans K, Goossens S, et al. The community case management of pneumonia in Africa: a review of the evidence. Health Policy Plan 2015;30(2):253-266. [doi: 10.1093/heapol/czt104]

14. Gardner AJ, Iverson GL, Quinn TN, et al. A preliminary video analysis of concussion in the National Rugby League. Brain Inj 2015;29(10):1182-1185. [doi: 10.3109/02699052.2015.1034179]

15. Gardner AJ, Iverson GL, Stanwell P, et al. A video analysis of use of the new 'concussion interchange rule' in the national rugby league. Int J Sports Med 2016;37(04):267-273. [doi: 10.1055/s-0035-1565203]

16. Hinton-Bayre AD, Geffen G, Friis P. Presentation and mechanisms of concussion in professional Rugby League football. J Sci Med Sport 2004;7(3):400-404. [doi: 10.1016/S14402440(04)80035-5]

17. Gardner AJ, Howell DR, Levi CR, et al. Evidence of concussion signs in national rugby league match play: a video review and validation study. Sports Med Open 2017;3(1):29. [doi: 10.1186/s40798-017-0097-9][PMID: 28831759]

18. Gardner AJ, Levi CR, Iverson GL. Observational review and analysis of concussion: a method for conducting a standardized video analysis of concussion in rugby league. Sports Med Open 2017;3(1):26. [doi: 10.1186/s40798-017-0093-0][PMID: 28710323]

19. Gardner AJ, Kholer RM, Levi CR, et al. Usefulness of video review of possible concussions in national youth rugby league. Int J Sports Med 2017;38(01):71-75. [doi: 10.1055/s-0042116072][PMID: 27737484]

20. Gardner AJ, Howell DR, Iverson GL. A video review of multiple concussion signs in National Rugby League match play. Sports Med Open 2018;4:5. [doi: 10.1186/s40798-017-01179][PMID: 29330738]

21. Carmody DJ, Taylor TK, Parker DA, et al. Spinal cord injuries in Australian footballers 1997-2002. Med J Aust 2005;182(11):561-564. [doi: 10.5694/j.1326-5377.2005.tb06814.x] [PMID: 15938682]]

22. McDonough A, Funk L. Can glenohumeral joint isokinetic strength and range of movement predict injury in professional rugby league. Phys Ther Sport 2014;15(2):91-96. [doi: 10.1016/j.ptsp.2013.07.001][PMID: 23948328]

23. Gissane $C$, Jennings $D$, White J, et al. Injury in summer rugby league football: the experiences of one club. Br J Sports Med 1998;32(2):149-152. [doi: 10.1136/bjsm.32.2.149][PMID: 9631223]

24. Gissane C, Jennings D, Kerr K, et al. Injury rates in rugby league football: impact of change in playing season. Am J Sports Med 2003;31(6):954-958. [doi: 10.1177/03635465030310063501] [PMID: 14623663]

25. Gissane C, White J, Kerr K, et al. Health and safety implications of injury in professional rugby league football. Occup Med 
(Lond) 2003;53(8):512-517. [doi: 10.1093/occmed/kqg103] [PMID: 14673125]

26. Gabbett TJ, Ullah S, Finch CF. Identifying risk factors for contact injury in professional rugby league players application of a frailty model for recurrent injury. J Sci Med Sport 2012;15(6):496-504. [doi: 10.1016/j.jsams.2012.03.017] [PMID: 22748762]

27. Georgeson EC, Weeks BK, McLellan C, et al. Seasonal change in bone, muscle and fat in professional rugby league players and its relationship to injury: a cohort study. BMJ Open 2012; 2(6):e001400. [doi: 10.1136/bmjopen-2012-001400][PMID: 23135539]

28. Sanctuary CE, Meir R, Sadler I. The seven step approach to the application of sports science in English professional rugby league: Practical considerations in strength and conditioning. Int J Sports Sci Coach 2012;7(1):33-45. [doi: 10.1260/1747-9541.7.1.33]

29. Ullah S, Gabbett TJ, Finch CF. Statistical modelling for recurrent events: an application to sports injuries. Br J Sports Med 2014;48(17):1287-1293. [doi: 10.1136/bjsports-2011090803][PMID: 22872683]

30. Babic Z, Misigoj-Duraković M, Matasić H, et al. Croatian rugby project. Part II: injuries. J Sports Med Phys Fitness 2001;41:392-398. [PMID: 11533572]

31. Gabbett TJ. Severity and cost of injuries in amateur rugby league: a case study. J Sports Sci 2001;19(5):341-347. [doi: 10.1080/02640410152006117][PMID: 11354613]

32. Gabbett $\mathrm{T}$, Hodgson P. Incidence of injury in semiprofessional rugby league players. Br J Sports Med 2003;37(1):36-44. [doi: 10.1136/bjsm.37.1.36][PMID: 12547741]

33. Gabbett TJ. Influence of training and match intensity on injuries in rugby league. J Sports Sci 2004;22(5):409-417. [doi: 10.1080/02640410310001641638][PMID: 15160594]

34. Gabbett TJ. Influence of playing position on the site, nature, and cause of rugby league injuries. J Strength Cond Res 2005;19(4):749-755. [doi: 10.1519/R-16504.1][PMID: 16287361]

35. Gabbett TJ. Influence of the limited interchange rule on injury rates in sub-elite Rugby League players. J Sci Med Sport 2005;8(1):111-115. [doi: 10.1016/S1440-2440(05)80031-3] [PMID: 15887908]

36. Gabbett TJ, Domrow N. Risk factors for injury in subelite rugby league players. Am J Sports Med 2005;33(3):428-434. [doi: 10.1177/0363546504268407]PMID: 15716260]

37. King DA, Gabbett TJ, Dreyer C, et al. Incidence of injuries in the New Zealand national rugby league sevens tournament. J Sci Med Sport 2006;9(1-2):110-118. [doi: 10.1016/j.jsams.2005.09.001][PMID: 16584918]

38. Gabbett TJ. Incidence of injury in junior rugby league players over four competitive seasons. J Sci Med Sport 2008;11(3):323-328. [doi: 10.1016/j.jsams.2007.06.003][PMID: 17698413]

39. King DA, Gissane C. Injuries in amateur rugby league matches in New Zealand: a comparison between a division 1 and a division 2 premier grade team. Clin J Sport Med 2009;19(4):277-281.

10.1097/JSM.0b013e3181a7c6b0][PMID: 19638820]

40. Orr R, Cheng HL. Incidence and characteristics of injuries in elite Australian junior rugby league players. J Sci Med Sport 2016;19(3):212-217. [doi: 10.1016/j.jsams.2015.03.007][PMID: 25882397]

41. Sirotic AC, Coutts AJ, Knowles H, et al. A comparison of match demands between elite and semi-elite rugby league competition. J Sports Sci 2009;27(3):203-211. [doi: 10.1080/02640410802520802][PMID: 19153858]

42. Gabbett TJ, Jenkins DG, Abernethy B. Relationships between physiological, anthropometric, and skill qualities and playing performance in professional rugby league players. J Sports Sci 2011;29(15):1655-1664. [doi: 10.1080/02640414.2011.610346] [PMID: 22092276]

43. McLellan CP, Lovell DI, Gass GC. Biochemical and endocrine responses to impact and collision during elite Rugby League match play. J Strength Cond Res 2011;25(6):1553-1562. [doi: 10.1519/JSC.0b013e3181db9bdd][PMID: 21602645]

44. Sirotic AC, Knowles H, Catterick C, et al. Positional match demands of professional rugby league competition. J Strength Cond Res 2011;25(11):3076-3087.

[doi: 10.1519/JSC.0b013e318212dad6] [PMID: 21993021]

45. Sykes D, Twist C, Nicholas C, et al. Changes in locomotive rates during senior elite rugby league matches. J Sports Sci 2011;29(12):1263-1271. [doi: 10.1080/02640414.2011.582507] [PMID: 21815859]

46. Duffield R, Murphy A, Snape A, et al. Post-match changes in neuromuscular function and the relationship to match demands in amateur rugby league matches. J Sci Med Sport 2012;15(3):238-243. [doi: 10.1016/j.jsams.2011.10.003][PMID: 22137196]

47. McLellan CP, Lovell DI. Neuromuscular responses to impact and collision during elite rugby league match play. J Strength Cond Res 2012;26(5):1431-1440.

[doi: 10.1519/JSC.0b013e318231a627][PMID: 22516913]

48. Twist C, Waldron M, Highton J, et al. Neuromuscular, biochemical and perceptual post-match fatigue in professional rugby league forwards and backs. J Sports Sci 2012;30(4):359367. [doi: 10.1080/02640414.2011.640707][PMID: 22176201]

49. Johnston RD, Gibson N V, Twist C, et al. Physiological responses to an intensified period of rugby league competition. J Strength Cond Res 2013;27(3):643-654.

[doi: 10.1519/JSC.0b013e31825bb469][PMID: 22592168]

50. McGuckin TA, Sinclair WH, Sealey RM, et al. The effects of air travel on performance measures of elite Australian rugby league players. Eur J Sport Sci 2014;14 (sup 1):S116-122. [doi: 10.1080/17461391.2011.654270]

51. Cummins C, Orr R. Analysis of physical collisions in elite National Rugby League match play. Int J Sports Physiol Perform 2015;10(6):732-739. [doi: 10.1123/ijspp.20140541][PMID: 25945899]

52. Fletcher BD, Twist C, Haigh JD, et al. Season-long increases in perceived muscle soreness in professional rugby league players: role of player position, match characteristics and playing surface. J Sports Sci 2016;34(11):1067-1072. [doi: 10.1080/02640414.2015.1088166][PMID: 26368285]

53. Speranza MJ, Gabbett TJ, Johnston RD, et al. Relationship between a standardized tackling proficiency test and matchplay tackle performance in semiprofessional rugby league players. Int J Sports Physiol Perform 2015;10(6):754-760. [doi: 10.1123/ijspp.2015-0044][PMID: 26115181]

54. Dempsey GM, Gibson NV, Sykes D, et al. Match demands of senior and junior players during international rugby league. J Strength Cond Res 2018;32(6):1678-1684.

[doi: 10.1519/JSC.0000000000002028][PMID: 28800006]

55. Woods CT, Robertson S, Sinclair WH, et al. A comparison of game-play characteristics between elite youth and senior Australian National Rugby League competitions. J Sci Med Sport 2018;21(6):626-630. [doi: 10.1016/j.jsams.2017.10.003] [PMID: 29074344]

56. Hendricks S, Till K, den Hollander S, et al. Consensus on a video analysis framework of descriptors and definitions by the Rugby Union Video Analysis Consensus group. Br J Sports Med 2020; 54(10):566-572. [doi: 10.1136/bjsports-2019101293][PMID: 32079603] 
57. Gabbett TJ. Physiological and anthropometric correlates of tackling ability in rugby league players. J Strength Cond Res 2009;23(2):540-548. [doi: 10.1519/JSC.0b013e31818efe8b] [PMID: 19197211]

58. Gabbett T, Ryan P. Tackling technique, injury risk, and playing performance in high-performance collision sport athletes. Int J Sport Sci Coach 2009;4(4):521-533. [doi: 10.1260/174795409790291402]

59. Gabbett T, Kelly J, Pezet T. Relationship between physical fitness and playing ability in rugby league players. J Strength Cond Res 2007;21(4):1126-1133. [doi: 10.1519/r-20936.1] [PMID: 18076242]
60. Gabbett TJ, Jenkins DG, Abernethy B. Correlates of tackling ability in high- performance rugby league players. J Strength Cond Res 2011;25(1):72-79.

[doi: 10.1519/JSC.0b013e3181ff506f][PMID: 21157385]

61. Speranza MJ, Gabbett TJ, Johnston RD, et al. Muscular strength and power correlates of tackling ability in semiprofessional rugby league players. J Strength Cond Res 2015;29(8):20712078. [doi: 10.1519/JSC.0000000000000897] [PMID: 26200016]

62. Speranza MJ, Gabbett TJ, Johnston RD, et al. Effect of strength and power training on tackling ability in semiprofessional rugby league players. J Strength Cond Res 2016;30(2):336343. [doi: 10.1519/JSC.0000000000001058][PMID: 26813629] 